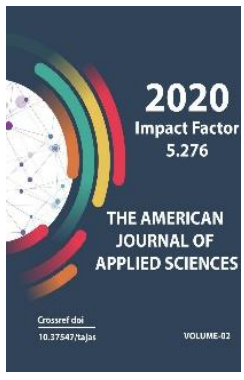

\section{Resolution Of The Effect Of Control On The Operation Of Other Machines In Opet-Pit Excavations}

\author{
Kodirov Vohid Rakhimovich \\ Associate Professor, Department of Mining, Tashkent City, 100095, Tashkent State Technical \\ University named after Islam Karimov, Uzbekistan
}

Journal Website: http://usajournalshub.c om/index,php/tajas

Copyright: Original content from this work may be used under the terms of the creative commons attributes 4.0 licence.

\title{
ABSTRACT
}

The article describes the method of influence of the explosion action on the stability of the instrument array in ore quarries during operation. The voltage of the instrument array from the explosion action on optically active models was studied. In technological processes, the stability of the sides of rock and semi-rock rocks and the value of the angle of inclination of the sides is most affected by drilling and blasting. To ensure the safety of the sides of deep quarries, we recommend the method and management of deformation processes. Using this method, it is possible to create a simulation of the pressure of detonation products on a static and dynamic setting.

\section{KEYWORDS}

Ledge, board, quarry, instrument array, stability, explosion, optical method, stress, modulus of elasticity, deformation, pressure, stress wave.

\section{INTRODUCTION}

Planning of deep quarries should be based on the study of engineering and geological conditions of quarry fields, the consequence of which is the zoning of the quarry according to the stability of ledges and sides in General. The main factors affecting the stability of 
rocks in the sides of quarries are revealed. The integrity of the ledges of the sides of the quarry depends primarily on the state of the instrument array, which is influenced by a number of factors. It is established that the stability of the sides of the quarry is directly related to natural and mining factors. The following natural factors are indicated: climatic conditions of the field, geological features, structural and tectonic factor, hydrogeological conditions of the field, the influence of earthquakes. The mining cluster includes the geometric parameters of the quarry, the impact of drilling and blasting operations, as well as the negative impact on the stability of mining transport.

As the depth of the quarry increases, the stability of the sides of the quarry becomes important. As the depth of the quarry increases, the risk of collapse of the sides of the quarry increases if the angles of repayment are incorrectly determined. On the other hand, an irrational reduction in the angles of the sides of the quarry causes the need for additional mining operations to separate the sides of the quarry. Thus when determining the stability of the sides of the quarry it is necessary to take into account the action of two limiting contradictory factors:

- creating safe working conditions that cause the sides of the quarry to be flattened;

- the desire to reduce the amount of work on additional spacing of the sides of the quarry, causing an increase in the angle of the slope of the side.

One of the main factors affecting the stability of the sides of the quarry is the nature of drilling and blasting operations. Generalizations and analysis of works performed in the field of studying the mechanism of action of an explosion in an array allow us to state that the destruction of solid media occurs under the combined action of gaseous detonation products, shock and elastic waves. Various research methods are used to study the effects of an explosion: theoretical, laboratory, and industrial.

For experimental methods, from the point of view of observing the similarity of gravity and studying the quasi-static effect of an explosion, the most acceptable method is the method of modeling an explosion in a lowmodulus gravitational model proposed by the A. A. Skochinsky IGD

To solve such problems, in practice, the polarization-optical method is widely used with the use of a device for pulsed pressure supply to charging chambers, which makes it possible to determine the dependence of deformation on the applied load.

Special studies conducted under laboratory conditions have shown that the distribution of maximum stresses under dynamic and static loads is graphically similar $[1,2,3]$.

\section{MATERIAL AND METHODS}

Received F. A. Belenko the study of the impact of the explosion optical method using highspeed filming, also matching the character of the stress distribution under static and dynamic loads.

As shown by the study on determining the ratio of the energy accounted for by the shock wave and the piston pressure of the explosion given in [3-4] and others by researchers, most of the explosion energy (70-80\%) is accounted for by the piston pressure.

Therefore, the results of the study on models obtained with static load application are suitable for determining the parameters of a 
dynamic process, in particular during blasting operations. This conclusion provides grounds for the effective use of the applied method for studying the stresses from the action of an explosion on optically active models.

Methods and techniques of experiments for solving various geomechanics problems by optical modeling are described in numerous works of G. N. Kuznetsov, F. A. Belaenko, V. F. Trumbechev, G. A. Katkov, G. A. Hesen, V. D. Morozov, V. R. Rakhimov, and others $[1,3,4,5,9]$

The brief essence of the photomechanics method is as follows. Optically sensitive isotropic materials under load are capable of exhibiting birefringence, the intensity of which is directly proportional to the value of the applied spiral. In the optically active model, instead of EXPLOSIVE charges, elastic chambers are placed to supply a pulse of compressed air. When pressure is applied to the chambers, the latter, expanding, affect the model, creating a picture of stress and deformation similar to that obtained from the EXPLOSIVE charge. The process of stress and strain development in the model is recorded from the initial to the final stage of the explosion development.

In most cases, flat models are used to study the mechanism of action of an explosion.

The theoretical justification for the use of flat models is quite fully described in the works of G. N. Kuznetsov, M. M. Budno and others [5]

\section{RESULTS}

The specifics of modeling the effect of an explosion on low-modulus optically active materials require investigation of the initial (before the action of the charge) stress fields in the model, taking into account all the necessary conditions for modeling a gravitating array with a cavity whose parameters correspond to the simulated charge structure.

Since the polarization-optical method of investigation is an experimental method of elasticity theory, the solution of the plane problem of elasticity theory is reduced to determining three components of stresses: $\sigma \mathrm{x}, \sigma \mathrm{u}, \sigma \mathrm{x}$, which must satisfy two differential equations of equilibrium and the compatibility equation under given boundary conditions. Provided that only gravity acts, the system of differential equations of equilibrium will have the form:

$$
\left.\begin{array}{l}
\frac{d \sigma_{x}}{d x}+\frac{d \tau_{x y}}{d y}=0 \\
\frac{d \sigma_{y}}{d y}+\frac{d \tau_{x y}}{d x}+y=0
\end{array}\right\}
$$

Since the weight of a unit of body volume $(y)$ is a constant value and does not depend on coordinates, the compatibility equation for a plane stress state of a plane strain will take the form:

$$
\left(\frac{d^{2}}{d x^{2}}+\frac{d \tau_{x y}}{d y^{2}}\right)\left(\sigma_{2}+\sigma_{y}\right)=0
$$

To determine the components of the voltage. $\sigma \mathrm{x}, \sigma \mathrm{u}, \sigma \mathrm{x}$ it is necessary to integrate the system of differential equations (1) and the compatibility equation (2) taking into account the conditions on the contour

$$
\begin{aligned}
& X_{n}=l \sigma_{x}+m \tau_{x y} \\
& Y_{n}=m \sigma_{y}+l \tau_{x y}
\end{aligned}
$$

where: $X$ and $Y$ are the components of the surface forces related to the area unit;

I, $m, n$ - the cosine guides are normal to the contour. The above equations show that 
under the conditions of the plane problem of elasticity theory for simply connected bodies and in the case of a material: $E$ (the elasticity model; and the Poisson's ratio) are absent and, therefore, do not affect the stress distribution in the medium under study (the Maurice-levy theorem).

The fulfillment of these conditions will ensure that in all elastic, homogeneous and isotropic geometrically similar bodies under equivalent loads the same type of stress distribution and the results of experimental studies obtained on optically active models can be transferred to real simulated objects.

In other words, the processes occurring in the model and the studied mountain range are considered similar if they have a geometric similarity and the similarity of the same physical variables that differ from each other by a constant multiplier.

The main forces that determine the main parameters of the medium under study are the acting forces and stresses. The defining similarity criteria based on Newton's General law of dynamic similarity and the dimensionality method for the problem of the joint action of gravitational forces and internal stresses are derived under the condition of known parameters of the forces acting in the medium under study;

$$
\frac{F}{f}=\frac{\gamma_{H}}{\gamma_{M}} \cdot \frac{l_{M}^{3}}{l_{M}^{3}}
$$

where: $F$ and $f$ are forces acting on identical points in nature and model

$\gamma_{H}, \gamma_{M}, I_{H}, I_{M}$ - volume weights and linear dimensions in the study area of nature and model,

If we denote

$$
\frac{f}{l_{M}^{2}}=N_{M} \quad \text { and } \quad \frac{F}{l_{\mu}^{2}}=N_{H}
$$

(5)

that's half the time

$$
\frac{N_{M}}{l_{M} \gamma_{M}}=\frac{N_{H}}{l_{H} \gamma_{H}}=\chi
$$

where: $\chi$-defining similarity criterion; $\mathrm{nm}, \mathrm{NH}$ values corresponding to various power characteristics having the stress dimension.

Formula (6) is considered the main similarity formula, provided that the relative deformations $(\varepsilon)$ and the Poisson's ratio $(\mu)$ of the model material and nature are equal. This formula can be represented as:

$$
\frac{E_{H}}{E_{M}}=\frac{\sigma_{H}}{\sigma_{M}}=\frac{\gamma_{H}}{\gamma_{M}} \cdot \frac{l_{H}}{l_{M}}
$$

If we provide the similarity of stresses, displacements in space, deformations of elementary volumes of the model and nature, then, in General, the presented model will be similar to the studied section of a stressed mountain range.

In the case of modeling the action of an explosive load, formula (6) is rewritten as:

$$
N_{M}=\frac{l_{M}^{2}}{l_{\mu}^{2}} \cdot \frac{t_{H}^{2}}{t_{M}^{2}} \cdot \frac{\rho_{M}}{\rho_{H}} \cdot N_{H}
$$

where: - the scale of densities;

$$
\frac{t_{H}}{t_{M}}=\alpha_{\rho} \text { - time scale. }
$$

The scale of characteristics (stresses, elastic modulus, taking into account the coefficient of inequality of relative deformations, pressures) is calculated by the formula: 


$$
\alpha_{H}=\alpha_{\rho} \frac{\alpha_{l}^{2}}{\alpha_{t}^{2}}
$$

$$
\text { or } \alpha_{\mu}=\alpha_{\rho} \cdot \alpha_{c p}^{2}
$$

In setting up the experiment, it is necessary to strictly take into account: the geometric scale that dictates the size of the model and the conditions for the convenience of constructing the model, the parameters of stress waves in nature, the time scale and elastic characteristics.

According to the simulation conditions, the geometric scale is selected from the possibility of constructing model charges and boundary conditions.

Usually in our research the geometric scale is accepted

The scale of stresses and elastic modulus is determined from the ratio:

$$
\alpha_{\sigma, E}=\frac{\sigma_{\max H}}{\sigma_{\max M}}=\frac{\sigma_{H}}{\sigma_{M}}=\frac{[\sigma]_{H}}{[\sigma]_{M}}=\frac{E_{H}}{E_{M}}
$$

The time scale depends on the geometric scale and the scale of the elastic propagation velocity in the model and in nature, i.e.,:

$$
\sigma_{t}=\frac{\alpha_{l}}{\alpha_{c p}}
$$

For example, if $\alpha_{e}=1 / 50, \alpha_{c p}=1 / 1000$, mo $\alpha_{t}=$ 20

\section{DISCUSSIONS}

Igdanite is mainly used as the model material, since its low modulus of elasticity and the possibility of wide variation of its properties by changing the material composition corresponds to the modeling condition in a given time interval of the impact of the pressure of detonation products on a nondestructible medium. The method allows you to conduct an experiment in both static and dynamic settings.

\section{REFERENCES}

1. Kadirov V. R. Method for assessing the impact of the explosion on the stability of the instrument array. / Ed. - Tashkent: Vestnik Tashstu., 1-2 / 2010. - articles 142143.

2. Snitka N. P., Nasirov U. F., Mislibaev I. T., Nutfulloev G. T. resource-Saving technologies for conducting drilling and blasting operations in quarries / Under the General editorship of Yu. d. Norov. Tashkent: Fan, 2017. - article 52.

3. Snitka N. P., Nasirov U. F., Umarov F. Ya., Zairov sh. sh. Control of parameters of drilling and blasting operations by stability of boards in deep quarries / Under the General editorship of Yu. d. Norov. Tashkent: Fan, 2017. - article 58.

4. Report on research work. Assessment of the geomechanical state of the " $M$ " quarry's instrument array taking into account influencing factors based on geodynamic zoning / / Tashstu. 2008. articles 45-46.

5. Guidelines for ensuring the stability of slopes and seismic safety of buildings and structures during blasting operations in quarries. - art. - Pb., 2007. - art. 17.

6. Kuznetsov G. V., Ulybin V. P. Deformation of the rock mass under the action of intense explosive loads. - GTS, 2009. - No. 2. - art. 32-35.

7. Instructions for monitoring deformations of sides, slopes of ledges and dumps in quarries and developing measures to ensure their stability. PB, 2005. - article 23.

8. Kuznetsov G. V., Ulybin V. P. Changes in the magnitude of the seismic impact of 
explosions at the quarry depending on various factors. - In sat.: Explosive case. M.: Nedra, 1992. - №71/28. - ST. 174-175.

9. Rakhimov V. R, Markov A.V., Kadirov V. R. study of the stresses of the Muruntau quarry instrument array taking into account tectonic forces. Gorny Vestnik of Uzbekistan, 2009, no. 4, articles 43-47.

10. Kadirov V. R. study of the stresses of the Muruntau quarry's instrument array taking into account tectonic forces. Herald of Tashkent State University, 2010. No. 1-2. article 141-146.

11. Kadirov V. R. Influence of the time factor on the state of the instrument array. Materials of the international scientific and technical conference. Navoi 2010. St 74-75

12. Kadirov V. R. assessment of the stability of the sides of deep horizons of the Muruntau quarry. Bulletin of The Tashkent State University, 2011. No. 4. article 47-51.

13. Махмудов Д.Р., Кадиров В.P. Assissment of the stability of quarry boards using the "Ustoi" program. South Asian Academic Research Journals. Indea. Impact Factor:7.13. 2020. st.919-926. 